\title{
Calidad morfológica de frutos y endocarpios del nanche rojo (Malpighia mexicana, Malpighiaceae)
}

\section{Morphological quality of fruits and endocarps of red nanche (Malpighia mexicana, Malpighiaceae)}

\author{
María de los Ángeles Maldonado Peralta,',6, Gabino García de los Santos², José Rodolfo García Nava², \\ Tarsicio Corona Torres ${ }^{4}$, Víctor Manuel Cetina Alcalá5 y Carlos Ramírez Herrera ${ }^{5}$
}

1 Universidad Autónoma de Guerrero, Unidad Académica Región de la Costa Chica, Licenciatura en Ciencia y Tecnología de Alimentos, Cruz grande, Municipio de Florencio Villareal, Guerrero, México.

2 Colegio de Postgraduados, Campus Montecillo, Programa en Recursos Genéticos y Productividad-Semillas, km. 36.5 carretera México-Texcoco, 56230 Edo. de México, México.

3 Colegio de Postgraduados, Campus Montecillo, Posgrado en Botánica, km. 36.5 carretera México-Texcoco, 56230 Edo. de México, México.

4 Colegio de Postgraduados, Campus Montecillo, Programa en Recursos Genéticos y Productividad-Genética, km. 36.5 carretera México-Texcoco, 56230 Edo. de México, México

5 Colegio de Postgraduados, Campus Montecillo, Posgrado Forestal, km. 36.5 carretera México-Texcoco, 56230 Edo. de México, México.

6 Autor para correspondencia: angelitam_02@hotmail.com

\section{Citar como:}

Maldonado Peralta, M. A., G. García de los Santos, J. R. García Nava, T. Corona Torres, V. M. Cetina Alcalá y C. Ramírez Herrera. 2016. Calidad morfológica de frutos y endocarpios del nanche rojo (Malpighia mexicana), Malpighiaceae. Acta Botanica Mexicana 117: 37-46.

Recibido: 24 de noviembre de 2014

Revisado: 6 de junio de 2016 .

Aceptado: 23 de agosto de 2016.

\section{Resumen:}

Antecedentes y Objetivos: El nanche rojo silvestre se desarrolla en climas templados y tropicales. En México, desde tiempos prehispánicos se ha utilizado como ornamental y medicinal; sin embargo, se conoce poco sobre su morfología. Por ello el objetivo de esta investigación fue caracterizar la calidad morfológica de frutos y endocarpios de nanche rojo, de los Valles Centrales de Oaxaca, México. Métodos: Se ubicaron tres zonas de estudio, pero solo en el municipio de Santiago Matatlán se encontraron frutos, los cuales fueron recolectados y trasladados al Laboratorio de Análisis de Semillas del Colegio de Postgraduados, Campus Montecillo. Para la evaluación de la calidad morfológica de frutos y endocarpios, se seleccionaron mediante un Diseño Completamente al Azar (DCA) cuatro repeticiones de 100 frutos. Con los datos obtenidos se realizó un análisis de medidas de tendencia central, utilizando el paquete estadístico SAS.

Resultados clave: Los resultados encontrados indicaron que los frutos del nanche rojo son grandes, tienen forma de oblato, el color del epicarpio es guinda a morado, el del mesocarpio rosa a lila, de sabor dulce, con adecuada firmeza y cantidad de ácido ascórbico. El fruto tiene tres endocarpios fibrosos, de forma cono-triangular, y de uno a dos presentan embrión. El embrión es de color crema y no posee endospermo, los cotiledones doblados están en el ápice y en el otro extremo se encuentra la radícula. Está protegido por un tegumento delgado color café.

Conclusiones: Se observó variación en las características morfológicas. Los frutos tienen potencial alimenticio y calidad de exportación.

Palabras clave: acerola, Malpighia emarginata, Malpighia glabra, Malpighia mexicana, Malpighia punicifolia, semeruco.

\section{ABSTRACT:}

Background and Aims: Wild red nanche develops in both temperate and tropical areas. In Mexico, it has been used as an ornamental and medicinal plant since pre-Hispanic times; however, little is known about its morphology. Therefore, the aim of this research was to characterize the morphological quality of fruits and endocarps of red nanche collected in the Central Valleys of Oaxaca, Mexico. Methods: Three study areas were located, but fruits were found in the municipality of Santiago Matatlán only. These were collected and transferred to the Seed Analysis Laboratory of the Colegio de Postgraduados, Campus Montecillo. For evaluation of the morphological quality of fruit and endocarps, four replications of 100 fruits were selected by Completely Randomized Design (CRD). With the obtained data, an analysis of measures of central tendency was performed using the SAS statistical package.

Key results: The results indicated that the fruits of red nanche are large, oblate-shaped, the color of the epicarp is cherry to purple, that of the mesocarp pink to lilac, they taste sweet, and show adequate firmness and quantity of ascorbic acid. The fruit has three fibrous endocarps, is cone to triangular-shaped and has one to two embryos. The embryo is cream colored without endosperm, the cotyledons are bent at the apex and the radicle is present at the other side. It is protected by a thin brown colored integument.

Conclusions: Variation was observed in the morphological characteristics. The fruits have food potential and quality for export.

Key words: acerola, Barbados Cherry, Malpighia emarginata, Malpighia glabra, Malpighia mexicana, Malpighia punicifolia. 


\section{INTRODUCCIÓN}

El nanche rojo (Malpighia mexicana A. Juss.), sinónimo de la acerola (Malpighia glabra L.) y el semeruco (Malpighia punicifolia L. o Malphigia emarginata D.C.), es originario del sur de México, América Central y de la zona septentrional de Sudamérica. La población nativa de las islas de América Central consume los frutos y dispersa las semillas (Couceiro, 1985; Nassif y Cícero, 2006). Los españoles nombraron estos frutos como "la cereza de las Indias Occidentales" por su color y sabor, parecidos a la cereza europea (Mezadri et al., 2006). Actualmente, se conocen como cereza colorada, cereza de barbados, cereza de las Antillas, manche, nanche, nanche rojo, acerola y semeruco (Mezadri et al., 2006). Malpighia emarginata se introdujo como ornamental a los Estados Unidos de América en 1880 por Pliny Reasoner y su uso como comestible se ha documentado desde 1903 (Ledin, 1958).

Este fruto tiene alta demanda por el contenido de vitamina $\mathrm{C}$, así como sus propiedades antioxidantes y nutrimentales (Dean et al., 1997; Hassimotto et al., 2005; Riguetto et al., 2005). La fructificación ocurre durante los meses de mayo a diciembre (Simão, 1971). Los frutos son climatéricos de maduración rápida (Alves, 1996) y cuando se cosechan en madurez fisiológica el deterioro en almacén se presenta a los cuatro o cinco días (Manica y Carvalho, 1995). En México, el nanche rojo silvestre se distribuye en Chiapas, Durango, Estado de México, Guerrero, Jalisco, Michoacán, Oaxaca, Puebla y Yucatán (Guízar y Sánchez, 1991; Juárez, 1998). Principalmente se usa como cerca viva, medicinal contra afecciones estomacales, diabetes, escorbuto y como alimento, entre otras (Morton, 1987). El arbusto es tolerante a la sequía, crece en suelos deficientes en nutrimentos y genera semillas con alta viabilidad (Maldonado-Peralta et al., 2016); sin embargo, las poblaciones silvestres han disminuido debido al aumento de zonas urbanas. Por lo anterior, la exploración etnobotánica, recolecta del germoplasma, caracterización, resguardo, cultivo y domesticación son necesarios, ya que éste puede aprovecharse como alternativa de cultivo en diferentes zonas tropicales del país (Jarquín, 2007).
Los frutos de acerola tienen alto contenido de ácido ascórbico (Itoo et al., 1990; Matsuura et al., 2001). Los frutos de nanche rojo tienen características morfológicas similares a las de acerola o semeruco (Yamashita et al., 2003; Antunes et al., 2006). Consecuentemente, se requiere realizar estudios de la composición química del nanche rojo, para iniciar la promoción de sus usos y bondades, que contribuyan al conocimiento e interés para su conservación. También se requieren investigaciones para determinar su distribución específica y variabilidad genética, de acuerdo a las condiciones edafo-climáticas donde prospera este frutal, para contribuir a la conservación del recurso fitogenético. Por otra parte, esta especie mexicana puede destinarse al mercado de exportación, para consumo en fresco o para la industria, ya que presenta contenido de Sólidos Solubles Totales (SST) superiores a los que exigen la industria (8\%) y los mercados de exportación (Europa 7\% y Japón 7.5\%) (Alves, 1996; Lopes y Pavia, 2002).

La información relativa al nanche rojo es escasa, sobre todo aquella que describe la morfología de frutos y semillas, aspectos de importancia taxonómica y de manejo (Von Teichman y Van Wyk, 1991). Por lo anterior, el objetivo de la presente investigación fue determinar la calidad morfológica de frutos y endocarpios de nanche rojo, recolectados en Santiago Matatlán, Oaxaca.

\section{Materiales y MÉtOdos}

La investigación se realizó en tres localidades de los Valles Centrales de Oaxaca: Santa María Zoquitlán, San Pedro Totolapan y Santiago Matatlán. Sin embargo, sólo se encontraron frutos en arbustos silvestres del municipio de Santiago Matatlán, en septiembre de 2013. Esta localidad se ubica a $16^{\circ} 51^{\prime} 39.28^{\prime \prime}$ de latitud Norte y $96^{\circ} 22^{\prime} 50.11^{\prime \prime}$ latitud Oeste, a una altitud de $1729 \mathrm{~m}$. El clima es cálido subhúmedo con lluvias en verano (INEGI, 2012). Los frutos se trasladaron al Laboratorio de Análisis de Semillas del Colegio de Postgraduados, Campus Montecillo, en donde se seleccionaron los sanos y completos. Posteriormente, fueron lavados con agua y secados a temperatura ambiente. 
Mediante un Diseño Completamente al Azar (DCA) se eligieron cuatro repeticiones de 100 frutos, para la obtención de los datos de la calidad morfológica. El número de frutos incluidos en la muestra se hizo con base en la disponibilidad en campo y considerando las características de diferentes ámbitos de variación morfológica y la distribución geográfica en Oaxaca. El diámetro polar y ecuatorial de los frutos se midieron con vernier (vernier Truper Stainless ${ }^{\circledR}$ Steel). El diámetro polar del fruto se determinó desde el extremo apical hasta la base. El diámetro ecuatorial se midió en la porción media del fruto. El índice de forma del fruto resultó de dividir el diámetro polar entre el diámetro ecuatorial (Alia-Tejacal et al., 2012). Se determinaron los colores del epicarpio y mesocarpio con un colorímetro (Chroma meter CR-400, Ramsey, EUA) que registra los valores de $L^{*}, a^{*}$ y $b^{*}$, reportados como luminosidad ( $\left.\mathrm{L}^{*}\right)$, ángulo de matiz $\left(\tan ^{-1} \mathrm{~b}^{*} / \mathrm{a}^{*}\right)$ y cromaticidad $\left(\sqrt{\left(a^{*}\right) 2+\left(b^{*}\right)^{2}}\left(\sqrt{\left(a^{*}\right) 2+\left(b^{*}\right)^{2}}\right)\right.$ (McGuire, 1992). El peso del fruto, peso fresco y seco del mesocarpio se obtuvieron con una balanza electro-analítica (Scientech ZSA120, Boulder, EUA). Los frutos se secaron en una estufa durante 72 horas a $70{ }^{\circ} \mathrm{C}$. Los Sólidos Solubles Totales (SST) se midieron con un refractómetro (HANNA instruments, HI 96801, Éiba, España), utilizando una gota de jugo del mesocarpio de cada fruto. La acidez titulable (AT) se evaluó mediante el método volumétrico, se procedió de la siguiente manera: a) se tomaron muestras de $10 \mathrm{~g}$ de mesocarpio y se molieron con agua destilada; b) el agua se filtró; c) se tomaron alícuotas de $5 \mathrm{ml}$; d) se agregaron dos gotas de fenolftaleína (1\%) a las alícuotas y se tituló con $\mathrm{NaOH} 0.1 \mathrm{~N}$. El índice de sabor se obtuvo al dividir los valores de SST y AT. Los análisis de ácido ascórbico se realizaron con el método de Tillman (AOAC, 1980). Los datos se reportaron en $\mathrm{g} / \mathrm{L}$ de jugo (AOAC, 1990). La firmeza se midió con un texturómetro universal (Force-Five. FDV-30, Greenwich, EUA) con precisión en Newton, considerando los parámetros designados por Folder (1986) para Fragaria spp.

La forma, color, peso, grosor del endocarpio, así como diámetro polar y ecuatorial de los endocarpios se midieron. Además, se evaluó el número de embriones por fruto y se calcularon la relación entre el peso de los embriones y el peso de los endocarpios. El color se determinó y el peso (g) se registró. El largo y ancho (mm) del embrión se midieron. El índice de forma se calculó a través de la relación diámetro polar entre diámetro ecuatorial. Los datos de las variables se analizaron con el procedimiento de medidas de tendencia central, utilizando el paquete estadístico SAS ${ }^{\circledR} 9.2$ (SAS Institute, 2009).

\section{Resultados y discusión}

\section{Calidad morfológica del fruto}

El peso de los frutos silvestres de nanche rojo osciló entre 6.96 y 8.08 g (Cuadro 1). Freire et al. (2006) reportaron valores tres veces más bajos (1.62 a $2.83 \mathrm{~g})$ en frutos cultivados de acerola (M. emarginata y M. punicifolia); sin embargo, el peso de los frutos de nanche rojo fue similar al de los frutos de acerola (Brunini et al., 2004; Silva, 2008). Los diámetros polar y ecuatorial variaron entre 19.54 y $25.05 \mathrm{~mm}$, datos que coincidieron con los valores reportados para acerola por França y Narain (2003), Freire et al. (2006) y Silva (2008). La forma de los frutos de nanche rojo silvestre, resultó de dividir el diámetro polar entre el ecuatorial (Alia-Tejacal et al., 2012), lo que les confirió forma de oblato (0.78), es decir son más largos (Diametro ecuatorial) que anchos (Diametro polar). Al respecto, las acerolas variaron de 41.93 hasta $93.88 \%$ de mesocarpio (Freire et al., 2008) y se consideran de calidad para consumo en fresco o procesado. El nanche rojo obtuvo en promedio $64.42 \%$ en relación al peso del fruto, de esta manera se afirma que los frutos son de calidad comercial y por lo tanto representan una alternativa económica para las zonas tropicales con bajo potencial productivo.

Los frutos del nanche rojo son climatéricos, presentaron diversidad en formas, color, aroma y brillo (Fig. 1). Estas características se pierden conforme los frutos maduran; la luminosidad ( $L^{*}$ ) en el epicarpio fue de 24.53 a 25.46. El mesocarpio tuvo mayor variabilidad, los valores oscilaron entre 21.61 y 32.27 ; la cromaticidad (C*) fue menor en el epicarpio (14.83) que en el mesocarpio (20.34); el matiz ( $\left.\mathrm{M}^{*}\right)$ tuvo el mayor valor y tiende a to- 
Cuadro 1: Descriptores de características cuantitativas y parámetros estadísticos de calidad de frutos de nanche rojo ( $M$. mexicana A. Juss.). $\mathrm{N}=400$; Rango=Rango de Variación; CV= Coeficiente de Variación; EE=Error Estándar.

\begin{tabular}{|c|c|c|c|c|c|c|}
\hline Variable & Mínimo & Máximo & Media & Rango & $\mathrm{CV}$ & $\mathrm{EE}$ \\
\hline Peso de fruto $(\mathrm{g})$ & 6.96 & 8.08 & 7.39 & 1.12 & 5.99 & 1.19 \\
\hline Peso de endocarpio fresco $(\mathrm{g})$ & 4.06 & 5.24 & 4.76 & 1.18 & 11.03 & 0.24 \\
\hline Peso de endocarpio fresco (g) & 0.62 & 0.84 & 0.73 & 0.22 & 12.41 & 0.04 \\
\hline Diámetro polar (mm) & 19.04 & 20.01 & 19.54 & 0.97 & 1.84 & 0.16 \\
\hline Diámetro ecuatorial (mm) & 24.32 & 25.91 & 25.03 & 1.59 & 2.41 & 0.27 \\
\hline \multicolumn{7}{|l|}{ Parámetros de calidad } \\
\hline \multicolumn{7}{|l|}{ Color del epicarpio } \\
\hline Luminosidad (L*) & 24.53 & 25.46 & 24.94 & 0.93 & 1.64 & 0.18 \\
\hline Cromaticidad $\left(\mathrm{C}^{*}\right)$ & 13.25 & 16.81 & 14.83 & 3.56 & 8.68 & 0.58 \\
\hline $\operatorname{Matiz}\left(\mathrm{H}^{*}\right)$ & 80.58 & 82.7 & 81.48 & 2.12 & 1.24 & 0.45 \\
\hline \multicolumn{7}{|l|}{ Color del mesocarpio } \\
\hline Luminosidad (L*) & 21.61 & 32.27 & 27.58 & 10.66 & 15.04 & 1.85 \\
\hline Cromaticidad $\left(\mathrm{C}^{*}\right)$ & 17.27 & 23.15 & 20.34 & 5.88 & 10.57 & 0.96 \\
\hline Matiz $\left(\mathrm{H}^{*}\right)$ & 74.81 & 87.67 & 81.04 & 12.86 & 5.92 & 2.15 \\
\hline Solidos solubles totales $\left({ }^{\circ}\right.$ Brix $)$ & 9.9 & 10.48 & 10.18 & 0.58 & 2.06 & 0.09 \\
\hline Acidez Titulable (\%) & 0.85 & 1.13 & 1.01 & 0.28 & 11.61 & 0.05 \\
\hline Índice de forma (Diámetro polar / Diámetro ecuatorial) & 0.77 & 0.79 & 0.78 & 0.02 & 0.91 & 0.03 \\
\hline Índice de sabor (SST/AT) & 9 & 11.62 & 10.23 & 2.62 & 11.04 & 0.51 \\
\hline Vitamina C (g) & 0.1 & 1 & 0.29 & 0.9 & 134.4 & 0.18 \\
\hline Firmeza (N) & 4.53 & 7.09 & 5.69 & 2.56 & 16.42 & 0.42 \\
\hline
\end{tabular}

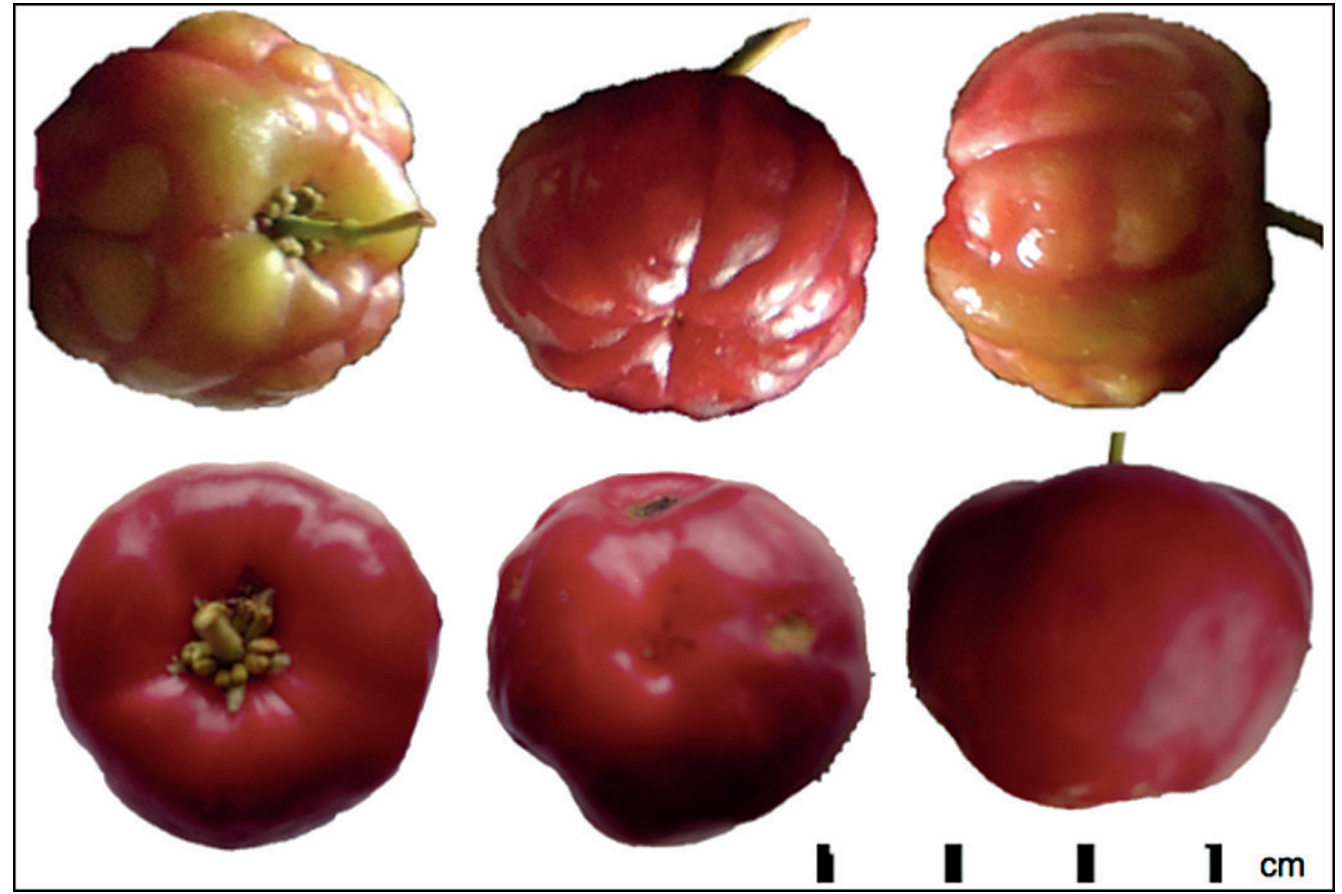

Figura 1: Frutos de nanche rojo (M. mexicana A. Juss.) en madurez fisiológica. 
mar un color claro tanto en el mesocarpio (81.04) como en el epicarpio (81.48). El color del epicarpio fue guindamorado brilloso. El mesocarpio varió entre blanco, rosa y lila, posiblemente como consecuencia de la presencia de algunas antocianinas, ya que Medrazi et al. (2008) reportaron diferentes cantidades de antocianinas del grupo de los flavonoides responsables del color en frutos maduros de acerola.

Los Sólidos Solubles Totales (SST) son otro atributo de calidad en el nanche rojo. Estos fluctuaron entre $9.9 \mathrm{y}$ $10.18^{\circ} \mathrm{Bx}$ (Brix); valores mayores a los obtenidos en acerola por Brunini et al. (2004) y Godoy et al. (2008), pero menores (11.97 y $\left.11.46^{\circ} \mathrm{Bx}\right)$ a los reportados por Freire et al. (2008) y Maciel et al. (2010). La fructosa, glucosa y pequeñas cantidades de sacarosa son los principales azúcares presentes en acerola (França y Narain, 2003); sin embargo, los valores de los azúcares totales en los frutos de nanche rojo pueden variar de acuerdo a la época del año, a la precipitación o a los procesos de degradación y biosíntesis de los polisacáridos (Kays, 1991). Como resultado se considera que esta especie mexicana puede ser utilizada para exportación o para la industria, porque sus valores de SST superaron a los que exige el mercado de exportación (Alves, 1996; Lopes y Pavia, 2002).

El ácido málico varió de 0.85 a $1.13 \%$ en $100 \mathrm{~g}^{-1}$ de pulpa (Cuadro 1). Valores equivalentes se encontraron en acerola ( 0.50 a $1.11,0.83$ a 1.35 y 0.69 a $1.65 \%)$ por Brunini et al. (2004), Godoy et al. (2008) y Matsuura et al. (2001), respectivamente, y mayores de hasta 1.97\% (Maciel et al., 2010).

Los ácidos orgánicos son los responsables de la acidez y el aroma particular de los frutos (Maciel et al., 2010); la acidez es una particularidad útil para conocer el estado de maduración de los frutos (Ladaniya, 2008). Esta característica tiene relación con los SST que determinan la singularidad del sabor (Ladaniya, 2008). El índice de sabor en los frutos maduros de nanche rojo varió de 9 a 11.62 , por lo que fueron relativamente deliciosos y dulces. Matsuura et al. (2001) encontraron un índice de 9.42 en acerola, mientras que França y Narain (2003) obtuvieron valores menores. Otros genotipos de acerolas alcanza- ron 7.06 de relación SST/AT (Maciel et al., 2010). Alves (1993) menciona que la proporción de SST/AT aumentó de 4 a 6.5 durante la maduración; estos atributos (SST/ AT) tienen como finalidad establecer el índice de cosecha y la selección de variedades para exportación, consumo de frutos en fresco y procesados (Couceiro, 1986; Kays, 1991).

El ácido ascórbico en los frutos de nanche rojo maduros varió de 100 a 1000 ppm. Los frutos de acerola contienen entre 1500 y $2360 \mathrm{mg}$ por $100 \mathrm{~g}$ de pulpa (Asenjo y Moscoso, 1950; Godoy et al., 2008). Estas concentraciones de ácido ascórbico son 100 veces mayores a las concentraciones que contiene la naranja (Todafruta, 2009). Los resultados de la presente investigación son similares a los que reportó Asenjo (1959). El contenido de vitamina $\mathrm{C}$ se relaciona con la altitud, ya que, a mayor altitud, menor es éste contenido (Asenjo, 1959). En frutos maduros, cuando el índice de sabor aumenta, el ácido ascórbico disminuye (Alves, 1996). Esto se puede deber a la relación entre la concentración de ácidos totales con el ácido ascórbico (Asenjo y Moscoso, 1950). Un genotipo con alta concentración de ácidos grasos y ácido ascórbico es difícil de obtener (Chitarra y Chitarra, 1990). Esta es la razón por la que la selección se direcciona hacia la obtención de genotipos sobresalientes en una de las dos características (Arostegui et al., 1954; Couceiro, 1986; Matsuura et al., 2001). La concentración de vitamina C es mayor cuando la precipitación es escasa (Simão, 1971). También depende de las prácticas culturales, luz, características genéticas, ubicación geográfica y edad de la planta (Nogueira et al., 2002).

La firmeza de los frutos de nanche rojo en madurez fisiológica varió de 4.53 a 7.09 N. Esto indica que se trata de frutos duros y firmes, lo que facilita el manejo. Folder (1986) menciona que valores ordinales entre 3 y 4 corresponden a frutos con firmeza y dureza; sin embargo, cuando los frutos de nanche rojo presentan madurez de consumo, cualquier presión o movimiento fuerte los daña, afecta la calidad y acelera el proceso de deterioro. Anderson (2000) menciona que cualquier fuerza externa superior a la soportada ocasiona cambios de sabor, color y aroma, por lo que 
existe una relación directa entre el tiempo de cosecha y la firmeza del fruto, dato que en nanche rojo se desconoce aún.

\section{Morfología del endocarpio}

El peso promedio de cada endocarpio fresco fue $0.92 \mathrm{~g}$, el peso seco de $0.39 \mathrm{~g}$, menos de la mitad del peso fresco (Cuadro 2). La longitud promedio fue $11.61 \mathrm{~mm}$, y la parte más ancha midió $8.79 \mathrm{~mm}$. Por su parte el fruto presentó tres endocarpios, como es el caso en el género Malpighia, cada uno con cavidad para un embrión, aunque sólo uno o dos embriones fueron viables. En acerola (M. emarginata) Nacif et al. (1996) y Nassif y Cícero (2006) encontraron de uno a dos endocarpios fértiles; esto puede obedecer a factores biológicos o genéticos, malformación o falta de fertilización del óvulo y degeneración del saco embrionario (Araújo y Minami, 1994; Azerêdo et al., 1994; Costa et al., 2003). Azerêdo et al. (1994), Nassif y Cícero, (2006) y Simplício et al. (1994) reportaron 29.4, 40 y 43\% de embriones normales, en 100 endocarpios evaluados, lo que coincide con lo encontrado en la presente investigación. Costa et al. (2003) indicaron que $51.33 \%$ de 300 endocarpios fueron normales.

Los endocarpios se clasificaron como fibrosos y de forma cono-triangular (Fig. 2). El color fue crema, con una luminosidad de 57.18; la cromaticidad y matiz fueron de 19.25 y 25.37 , respectivamente, debido a que las fibras de la testa se tiñen con el color del mesocarpio.

El peso y longitud promedio del embrión fue $0.02 \mathrm{~g} \mathrm{y}$ $6.59 \mathrm{~mm}$, la parte media presentó una anchura de $4.42 \mathrm{~mm}$, es aplanado, recto. Tiene un extremo de forma elíptica donde el ápice de los cotiledones se encuentra doblado hacia un lado del embrión, y en el tegumento se marca el rafe y la calaza como una hendidura pequeña, en forma de círculo acorazonado, color café oscuro, que se une al otro extremo del embrión donde se encuentra la radícula en forma cónica. El embrión es frágil, de color crema a amarillo, cubierto con un tegumento muy delgado color café. Las especies de la familia Malpighiaceae no presentan endospermo, pero tienen cotiledones grandes, bien desarrollados donde acumulan la reserva energética (Souto y Oliveira, 2008).

Cuadro 2: Descriptores de caracteres cuantitativos y parámetros estadísticos de calidad del endocarpio y embrión de nanche rojo (M. mexicana A. Juss.). $\mathrm{N}=400$; Rango=Rango de Variación; $\mathrm{CV}=$ Coeficiente de Variación; EE=Error Estándar.

\begin{tabular}{|c|c|c|c|c|c|c|}
\hline Variable & Mínimo & Máximo & Media & Rango & $\mathrm{CV}$ & $\mathrm{EE}$ \\
\hline \multicolumn{7}{|l|}{ ENDOCARPIO } \\
\hline Peso húmedo (g) & 0.71 & 1.04 & 0.92 & 0.33 & 14.88 & 0.06 \\
\hline Peso seco al ambiente $(\mathrm{g})$ & 0.37 & 0.42 & 0.39 & 0.05 & 5.37 & 0.01 \\
\hline Diámetro polar $(\mathrm{mm})$ & 10.92 & 12.41 & 11.61 & 1.49 & 4.57 & 0.24 \\
\hline Diámetro ecuatorial (mm) & 8.32 & 9.46 & 8.79 & 1.14 & 4.71 & 0.19 \\
\hline Número de embriones viables por fruto & 1.13 & 1.86 & 1.42 & 0.73 & 19.70 & 0.13 \\
\hline Peso del endocarpio $(\mathrm{g})$ & 0.09 & 0.14 & 0.11 & 0.05 & 19.57 & 0.01 \\
\hline Índice de forma (Diámetro polar / Diámetro ecuatorial) & 1.31 & 1.34 & 1.32 & 0.03 & 0.93 & 0.05 \\
\hline \multicolumn{7}{|l|}{ Color de endocarpio } \\
\hline Luminosidad $\left(\mathrm{L}^{*}\right)$ & 55.53 & 59.79 & 57.18 & 4.26 & 2.91 & 0.74 \\
\hline Cromaticidad $\left(\mathrm{C}^{*}\right)$ & 18.22 & 20 & 19.25 & 1.78 & 4.69 & 0.40 \\
\hline $\operatorname{Matiz}\left(\mathrm{H}^{*}\right)$ & 23.22 & 27.48 & 25.37 & 4.26 & 7.74 & 0.88 \\
\hline \multicolumn{7}{|l|}{ EMBRIÓN } \\
\hline Peso $(g)$ & 0.02 & 0.03 & 0.02 & 0.01 & 20.33 & 0.002 \\
\hline Diámetro polar (mm) & 6.48 & 6.66 & 6.59 & 0.18 & 1.03 & 0.030 \\
\hline Diámetro ecuatorial (mm) & 4.08 & 4.41 & 4.22 & 0.33 & 2.85 & 0.050 \\
\hline Índice de forma (Diámetro polar / Diámetro ecuatorial) & 1.51 & 1.62 & 1.56 & 0.11 & 2.74 & 0.010 \\
\hline
\end{tabular}




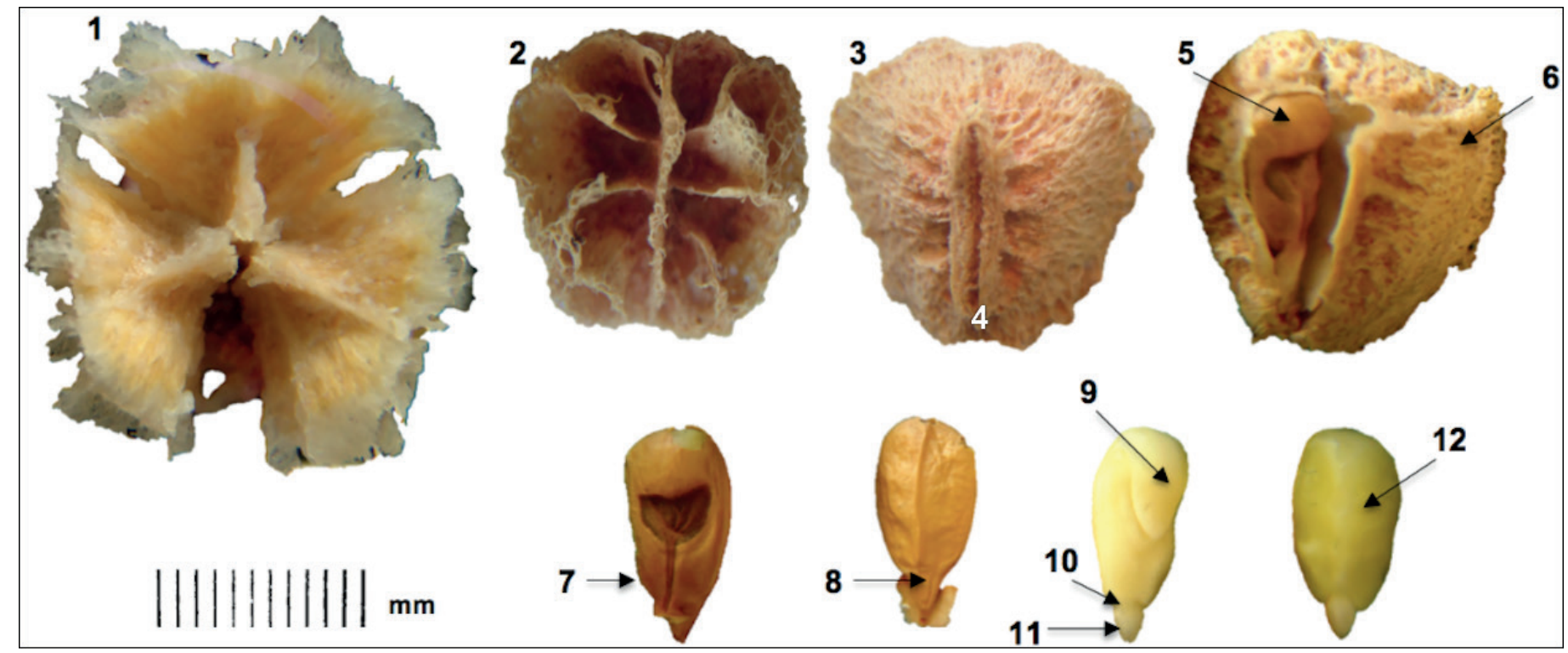

Figura 2: Semilla de nanche rojo (M. mexicana A. Juss.). 1. endocarpios dispuestos y que forman parte del fruto; 2. vista posterior; 3. vista frontal; 4. hilo; 5. posición del embrión del endocarpio; 6. testa; 7. rafe y calaza; 8. tegumento; 9. vista frontal con cotiledones doblados; 10. hipocótilo; 11. radícula; 12. vista posterior del embrión.

\section{CONCLUSIONES}

Los frutos del nanche rojo silvestre presentan mejores cualidades en tamaño y calidad (color, índice de sabor: SST/AT, firmeza, etc.) para consumo en fresco en el mercado nacional y de exportación, que la acerola cultivada.

El fruto varió en cuanto a forma, color del mesocarpio y concentración de azúcar para consumo en fresco, lo que debe homogeneizarse para facilitar su comercialización.

El índice de sabor en nanche rojo fue mejor que en la acerola y la acidez titulable similar. Lo anterior hace que esta especie presente potencial alimenticio y comercial, pero se requiere mayor investigación para conocer las propiedades nutrimentales, cultivarla de manera extensiva y contribuir a su domesticación.

\section{LITERATURA CITADA}

Alia-Tecajacal, I., Y. I. Astudillo-Maldonado, C. A. NúñezColín, L. A. Valdez-Aguilar, S. Bautista-Baños, E. García-Vazquez, R. Ariza-Flores y F. Rivera-Cabrera. 2012. Caracterización de frutos de ciruela mexicana (Spondias purpurea L.) del sur de México. Revista Fitotecnia Mexicana 35(5): 21-26.

Alves, R. E. 1993. Acerola (Malpighia emarginata D.C.): fisiologia da maturação armazenamento refrigerado sob atmosfera ambiente modificada. Tesis de maestría. Escuela Superior de Agricultura de Lavras (ESAL). Lavras, Brasil. 99 pp.

Alves, R. E. 1996. Características das frutas para exportação. In: Netto, G. A., E. F. G. Ardito y E. E. C. Garcia. (eds.). Acerola para exportação: procedimentos de colheita e pós-colheita. Brasilia, DF., Brasil. 21 pp.

Anderson, T. L. 2000. Fracture mechanics: Fundamentals and applications. 3rd ed. CRC Press. Inc. Boca Ratón, USA. $640 \mathrm{pp}$.

Antunes, A. M., J. Valmórbida, E. O. Ono y J. D. Rodrigues. 2006. Uso de reguladores vegetais na conservação refrigerada de acerolas (Malpighia glabra L.). Ciência e Agrotecnologia 30(6): 1241-1245. DOI: http://dx.doi. org/10.1590/S1413-70542006000600031.

AOAC. 1980. Official Methods of Analysis. Horwitz, W. (ed). $13^{\text {th }}$ ed. Association of Official Analytical Chemists. Washington, DC., USA. 1018 pp. 
AOAC. 1990. Fruits and fruits products. In: Helrich, K. (ed.). Official methods of analysis. Volume $1.15^{\text {th }}$ ed. Washington, D.C., USA. pp. 391-418.

Araújo, P. S. R. y K. Minami. 1994. Acerola. Fundação Cargill. Campinas, Brasil. 81 pp.

Arostegui, F., C. F. Asenjo, A. I. Muñiz y L. Alemañy. 1954. Studies on the West Indian Cherry, Malpighia punicifolia L.; observation and data on a promising selection. Proceedings of Florida State Horticultural Society 67: 250-255.

Asenjo, C. F. 1959. Aspectos químicos y nutritivos de la acerola (Malpighia punicifolia L.). Revista hispano-americana de Ciencias puras y aplicadas 19(6/7): 109-118.

Asenjo, C. F. y C. G. Moscoso. 1950. Ascorbic acid content and other characteristics of the West Indian Cherry. Journal of Food Science 15(2): 103-106.

Azerêdo, G. A., V. P. Matos., M. L. A. R. Germano y A. A. Lima. 1994. Efeito da temperatura e períodos de embebição na germinação de sementes de acerola (Malpighia glabra L.). In: XIII Congreso Brasileiro de Fruticultura. Salvador de Bahía, Brasil. p. 68-69.

Brunini, M. A., B. N. Macedo, V. C. Coelho y F. G. Siqueira. 2004. Caracterização física e química de acerolas provenientes de diferentes regiões de cultivo. Revista Brasileira de Fruticultura 26(3): 486-489. DOI: http://dx.doi. org/10.1590/S0100-29452004000300027

Chitarra, M. I. F. y A. B. Chitarra. 1990. Pós-colheita de frutos e hortaliças: fisiologia e manuseio. MG: ESAL/FAEPE (CPF-RR). Lavras, Brasil. 320 pp.

Costa, L. C., M. C. D. M. Do Pavani, F. V. Moro y D. Perecin. 2003. Viabilidade de sementes de acerola (Malpighia emarginata D.C.): avaliação da vitalidade dos tecidos. Revista Brasileira de Fruticultura 25(3): 532-534. DOI: http://dx.doi.org/10.1590/S010029452003000300043

Couceiro, E. M. 1985. Curso de extensão sobre a cultura da acerola. Recife: Universidade Federal Rural de Pernambuco, Brasil. 45 pp.

Couceiro, E. M. 1986. Acerola (Malpighia glabra L.): fabulosa fonte de vitamina $\mathrm{C}$ natural. In: X Reunião nordestina de botânica. Río Grande do Norte, Brasil.
Dean, R. T., S. Fu, R. Stocker y M. J. Davies. 1997. Biochemistry and pathology of radical-mediated protein oxidation. Biochemical Journal 324: 1-18.

Folder, F. 1986. La frutilla o fresa. Estudio de la planta y su producción comercial. Ed. Hemisferio Sur. Buenos Aires, Argentina. $150 \mathrm{pp}$.

França, V. C. y N. Narain. 2003. Caracterização química dos frutos de três matrizes de acerola (Malpighia emarginata D.C.). Food Science and Technology 23(2): 157-160. DOI: http://dx.doi.org/10.1590/S0101-20612003000200009

Freire, J. L. O., N. A. Lima, B. G. F. Santos y L. M. J. V. Marinus. 2006. Características físicas de frutos de acerola cultivada em Pomares de diferentes microrregiões do estado da Paraíba. Revista Agropecuária Técnica 27(2): 105-110.

Freire, J. L. O., N. A. Lima, O. A. L. Freire, M. J. V. Marinus, J. T. Dias y P. J. Silva. 2008. Avaliações biométricas de aceroleira (Malpighia emarginata D.C.) e caracterização dos atributos externos e internos dos frutos. Engenharia Ambiental: Pesquisa e Tecnologia 5(2): 41-52.

Godoy, R. C. B., E. L. S. Matos, T. S. Amorin, M. A. Souza Neto, R. Ritzinger y J. Waszczynsky. 2008. Avaliação de genótipos e variedades de acerola para consumo in natura e para elaboração de doces. Boletim do Centro de Pesquisa de Processamento de Alimentos 26(2): 197-204. DOI: http://dx.doi.org/10.5380/cep.v26i2.13274

Guízar, N. E. y V. A. Sánchez. 1991. Guía para el reconocimiento de los principales árboles del Alto Balsas. Universidad Autónoma de Chapingo. Chapingo, México. 207 pp.

Hassimotto, N. M. A., M. I. Genovese y F. M. Lajolo. 2005. Antioxidant Activity of Dietary Fruits, Vegetables, and Commercial Frozen Fruit Pulps. Journal Agriculture Food Chemistry 53(8): 2928-2935.

INEGI. 2012. Dirección General de Geografía. Coordinación de Desarrollo de Proyectos. Subdirección de Actualización de Marco Geoestadístico. Instituto Nacional de Estadística y Geografía. http://www.inegi.org.mx

Itoo, S., M. Aiba y K. Ishihata. 1990. Ascorbic acid content in acerola fruit from different production regions and degrees of maturity, and stability during processing. Journal of Japanese Society of Food Science and Technology 37(9): 726-729. 
Jarquín, L. R. 2007. Parasitoides asociados a insectos en frutos de nanche rojo (Malpighia mexicana) en Oaxaca. Tesis de maestría. Centro Interdisciplinario de Investigación para el Desarrollo Integral Regional, Instituto Politécnico Nacional, Unidad Oaxaca. Oaxaca, México. 53 pp.

Juárez, D. J. C. 1998. La familia Malpighiaceae en el estado de Morelos. Tesis de licenciatura. Facultad de Ciencias Biológicas, Universidad Autónoma del Estado de Morelos. Cuernavaca, México. 90 pp.

Kays, S. J. 1991. Postharvest physiology of perishable plant products. Van Nostrand Reinhold. New York, USA. 532 pp.

Ladaniya, S. M. 2008. Citrus fruits. Biology, Technology and Evaluation. Academic Press. San Diego, USA. 576 pp.

Ledin, R. B. 1958. The Barbados or West Indian cherry. Bulletin 594. University of Florida, Agricultural Experiment Station. Gainesville, USA. pp. 15-17.

Lopes, R. y J. R. Paiva. 2002. Aceroleira. In: Bruckner, C. H. (ed.). Melhoramento de fruteiras tropicais. Universidad Federal de Viçosa. Viçosa, Brasil. pp. 63-99.

Maciel, S. M. I., E. Melo, V. Lima, A. K. Souza y W. Silva. 2010. Caracterização físico-química de frutos de genótipos de aceroleira (Malpighia emarginata D.C.). Ciência e Tecnologia de Alimentos 30(4): 865-869.

Maldonado-Peralta, M. A., G. García de los Santos, J. R. García-Nava, C. Ramírez-Herrera, A. Hernández-Livera, J. M. Valdez-Carrazco, T. Corona-Torres y V. M. CetinaAlcalá. 2016. Seed viability and vigour of two nanche species (Malpighia mexicana and Byrsonima crassifolia). Seed Science and Technology 44(1): 168-176. DOI: http://dx.doi.org/10.15258/sst.2016.44.1.03

Manica, I. y R. I. N. Carvalho. 1995. Acerola, pesquisa e extensão no Rio Grande do Sul. In: São José, A. R. e R. E Alves (eds.). Acerola no Brasil: produção e mercado. Universidad Estadual de Sudoeste de Bahía, campus Vitória da Conquista. Bahía, Brasil. pp. 133-141.

Matsuura, F. C. A. U., R. L. Cardoso, da M. I. S. Folegatti, P. J. R. Oliveira, J. A. B. de Oliveira y D. B. Dos Santos. 2001. Avaliações físico-químicas em frutos de diferentes genótipos de acerola (Malpighia punicifolia L.). Revista Brasileira Fruticultura 23(3): 602-606. DOI: http://dx.doi. org/10.1590/S0100-29452001000300032.
McGuire, R. G. 1992. Reporting of objective color measurements. HortScience 27(2): 1254-1255.

Medrazi, T., D. Villaño, M. S. Fernández-Pachón, M. C. García-Parrilla and A. M. Troncoso. 2008. Antioxidant compounds and antioxidant activity in acerola (Malpighia emarginata D.C.) fruits and derivatives. Journal of Food Composition and Analysis 21(4): 282-290. DOI: http:// dx.doi.org/10.1016/j.jfca.2008.02.002

Mezadri, T., M. S. Fernández-Pachón, D. Villaño, M. C. García-Parrilla y A. M. Troncoso. 2006. El fruto de la acerola: composición, características productivas e importancia económica. Archivos Latinoamericanos de Nutrición 56(2) : 101-109.

Morton, J. F. 1987. Barbados Cherry In: Fruits of warm climates. Creative Resource Systems. Miami, USA. pp. 204207.

Nacif, R. S., C. M. Guardia y R. P. L. de Moraes. 1996. Morfología e anatomía das sementes de acerola (Malpighia glabra L.-Malpighiaceae). Revista Ceres 43(249): 597-610.

Nassif, P. D. S. y M. S. Cícero. 2006. Avaliação de sementes de acerola por meio de raios-X. Revista Brasileira de Fruticultura 28(3): 542-545.

Nogueira, R. J. M. C., J. A. P. V. de Moraes, H. A. Burity y J. F. da Silva Junior. 2002. Efeito do estádio de maturação dos frutos nas características físico-químicas da acerola. Pesquisa Agropecuaria Brasileira 37(4): 463-470.

Riguetto, A. M., F. M. Netto y F. Carraro. 2005. Chemical composition and antioxidant activity of juices from mature and immature acerola (Malpighia emarginata D.C.). Food Science and Technology International 11(4): 315-321.

SAS Institute. 2009. SAS/STAT ${ }^{\circledR}$ 9.2. User's Guide. SAS Institute. Cary, NC, USA. 1848 pp.

Silva, W. S. 2008. Qualidade e atividade antioxidante em frutos de variedades de aceroleira. Tesis de maestría. Centro de Ciências Agrárias, Curso de Pós-Graduação em Tecnologia de Alimentos. Universidade Federal de Ceará. Fortaleza, Brasil. 137 pp.

Simão, S. 1971. Cereja das Antilhas. Manual de Fruticultura. Editora Agronômica Ceres. São Paulo, Brasil. pp. 477-485.

Simplício, J. B., A. K. S. Silva, U. S. Souza Júnior, W. Y. Okasaki y R. S. Musser. 1994. Avaliação da presença de 
embrião em sementes de duas seleções de acerola (Malphighia glabra L.) na zona da Mata de Pernambuco. In: XIII Congresso Brasileiro de Fruticultura. Salvador de Bahía, Brasil. 81 pp.

Souto, S. L. y T. D. M. Oliveira. 2008. Morfoanatomia e ontogênese das sementes de espécies de Banisteriopsis C.B. Robinson e Diplopterys A. Juss. (Malpighiaceae). Acta Botanica Brasilica 22(3): 733-740. DOI: http://dx.doi. org/10.1590/S0102-33062008000300011.

Todafruta. 2009. Características de la acerola. Disponible en: www.todafruta.com.br. Fecha de consulta: julio de 2014.
Von Teichman, I. y A. E. Van Wyk. 1991. Trends in the evolution of dicotyledonous seeds based on character associations, with special reference to pachychalazy and recalcitrance. Botanical Journal of the Linnean Society 105(3): 211-237. DOI: http://onlinelibrary.wiley.com/ doi/10.1111/j.1095-8339.1991.tb00205.x/abstract

Yamashita, F., M. de T. Benassi, A. C. Tonzar, S. Moriya y J. G. Fernandes. 2003. Produtos de acerola: estudo da estabilidade de vitamina C. Ciência e Tecnologia de Alimentos 23(1): 92-94. DOI: http://dx.doi.org/10.1590/S010120612003000100019 